\title{
A Generative Adversarial Inpainting Network to Enhance Prediction of Periodontal Clinical Attachment Level
}

\section{Vasant Kearney ( $\nabla$ vasantkearney@gmail.com )}

Retrace Labs

Alfa-lbrahim M. Yansane

University of California, San Francisco

Ryan G. Brandon

University of California, San Francisco

Ram Vaderhobli

University of California, San Francisco

Guo-Hao Lin

University of California, San Francisco

Hamid Hekmatian

Retrace Labs

Wenxiang Deng

Retrace Labs

Neha Joshi

University of California, San Francisco

Harsh Bhandari

University of California, San Francisco

Ali S. Sadat

Retrace Labs

Joel M. White

University of California, San Francisco

\section{Research Article}

Keywords: Deep learning algorithms, clinical attachment levels (CAL), generative adversarial inpainting network, periodontal

Posted Date: December 10th, 2021

DOI: https://doi.org/10.21203/rs.3.rs-1094512/v1 
License: (c) (i) This work is licensed under a Creative Commons Attribution 4.0 International License. Read Full License 


\section{Abstract}

Deep learning algorithms has recently been used to determine clinical attachment levels (CAL) which aid in the diagnosis of periodontal disease. However, the limited field-of-view of dental bitewing x-rays poses a challenge for convolutional neural networks (CNN) because out-of-view anatomy cannot be directly considered. This study presents an inpainting algorithm using generative adversarial networks (GANs) coupled with partial convolutions to predict out-of-view anatomy to enhance CAL prediction accuracy. 80,326 images were used for training, 12,901 images were used for validation and 10,687 images were used to compare non-inpainted methods to inpainted methods for CAL predictions. Statistical analyses were conducted using mean bias error (MBE), mean absolute error (MAE) and Dunn's pairwise test comparing CAL at $p=0.05$. Comparator $p$-values demonstrated statistically significant improvement in CAL prediction accuracy between corresponding inpainted and non-inpainted methods with a MAE of $1.04 \mathrm{~mm}$ and $1.50 \mathrm{~mm}$ respectively. The Dunn's pairwise test indicated a statistically significant improvement in CAL prediction accuracy between both inpainted methods compared to their noninpainted counterparts, with the best performing methods achieving a Dunn's pairwise value of -63.89. This study demonstrates the superiority of using a generative adversarial inpainting network with partial convolutions to predict CAL from bitewing images.

\section{Introduction}

Periodontitis causes the loss of the gingival tissues and bone support around the teeth.(1,2) CAL is defined as the distance from the cemento-enamel junction to the base of the gingival sulcus during periodontal diagnostic probing. Mean annual loss of CAL of $0.1 \mathrm{~mm}$ per year has been reported in a general population.(3) In patients who have high susceptibility to periodontal disease, the loss of CAL can be as fast as $0.1-1 \mathrm{~mm}$ per year.(4) Therefore, identifying the change of CAL longitudinally is crucial to monitor disease progression. Radiographic anatomical localization has been utilized in the quantitative assessment and structural identification of dental anatomy(5). Segmentation accuracy by manual delineation is reliant on an individual's experience, subjective judgement, and is time consuming. Currently, no methods of automatic assessment of CAL are well developed and validated. Further, oral health care providers demonstrate calibration in their measurement of CAL, with recordings plus or minus $1 \mathrm{~mm}$, the increment able to be discerned by the clinician.

CAL is often verified using bitewing and periapical radiographs. Bitewings offer high contrast, high resolution, minimal spatial distortion, and relatively low radiation dose. Bitewings have a limited field of view (FOV) and might not capture enough maxillary or mandibular bone if compromised by image acquisition setup error, abnormal anatomy, or large patient anatomy.

To fill in missing image content, statistical image inpainting methods such as PatchMatch have been used $(6,7)$. These methods iteratively search for the best fitting patch but has no semantic awareness and can produce artifacts in the presence of heterogenous intensity information. Convolutional neural networks (CNNs) have been used in medical and dental applications to predict anatomy, radiation dose, 
image registration, periodontal health, and tooth labels(8-12). Conventional CNNs rely on pixel-level loss which encourages overly smooth predictions(13-15). To overcome this, conditional generative neural networks (GANs) have been engineered to encourage realistic predictions(16). GANs are comprised of at least one CNN generator and one CNN discriminator, trained adversarially to enforce image-level realism(17). GANs are difficult to train and computationally expensive because they rely competing CNNs, so the generators and discriminators are usually minimized to facilitate model convergence and accommodate for hardware limitations $(18,19)$. GANs have been applied to image inpainting in other domains but tend to produce artifacts when used on the periphery of images or on irregular shapes(20).

To solve this problem, a novel dental inpainting algorithm has been developed that uses a generative adversarial network combined with partial convolutions to increase the accuracy of CAL predictions from bitewing radiographs.

\section{Methods And Materials}

\section{Data Collection}

Permission to carry out this study, with waiver of consent and in compliance with HIPAA, was obtained from the UCSF Institutional Review Board (approval 18-24659) and all research was carried out in accordance with relevant guidelines and regulations. This study was a retrospective study, for which the requirement for informed consent was waived by the Institutional Review Board of UCSF due to its data source and methods. The data used for this study is not publicly available due to sensitive medical information, but is available from the corresponding author on reasonable request. CAL measurements were aggregated on patients spanning a three-and-a-half-year period from July 2016 through January 2020. Cases were matched with radiographic images (bitewing and periapical radiographs) acquired within 6 months prior to the periodontal therapy. Cases where periodontal charted values and diagnosis did not match with therapy were excluded as well as negative CAL values and those of $6 \mathrm{~mm}$ and above to maintain high ground truth data integrity. Purposive sampling selection criteria and cases selected were reviewed by 3 experienced academic practicing clinicians (one periodontist, GL 11 years of experience and two general dentists, RV 22 years of experience and JMW 38 years of experience) to verify ground truth data.

\section{Generative Adversarial Inpainting with Partial Convolutions}

The inpainting network is comprised of 2 generators and 3 discriminator CNNs. The Partial Convolution Encoder-Decoder generator focuses the network on missing regions of the images and fills in missing anatomy, while the second Refine Encoder-Decoder generator encourages overall realism of the image and helps refine the predictions from the Partial Convolutional Encoder-Decoder generator. The discriminators utilized a pre-trained VGG network and 1 patchGAN dynamic discriminator. Figure 1 depicts the information flow of the inpainting process as well as the various network components.

The Partial Convolutional Encoder-Decoder generator is comprised of 12 partial convolutional blocks that consist of $3 \times 3$ partial convolutional layers paired with leaky rectified linear unit (LeakyReLU) activation 
and synchronized instance normalization. The encoder stage utilized 6 Partial Convolutional blocks with stride 2 and the decoder stage utilized convolutional kernels with $2 x$ upsampling layers. The outputs of each multi-scale level in the encoding stage are concatenated with the upsampled corresponding decoding multi-scale level as part of the Partial Convolutional apparatus.

The Refine Encoder-Decoder generator consists of $27 \times 7$ convolutional blocks, and 7 residual blocks. The $7 \times 7$ convolutional blocks utilized $7 \times 7$ stride 2 convolutional kernels, synchronized instance normalization, $3 \times 3$ convolutional kernels, synchronized instance normalization, and ReLU activation. The residual blocks contained a $3 \times 3$ convolutional kernel, instance normalization, followed by a $3 \times 3$ convolutional kernel, instance normalization, and a residual connection that additively combined the input of the residual block with the output.

The VGG discriminator consisted of $54 \times 4$ convolutional blocks with instance normalization and a final average pooling layer. The $2 \mathrm{nd}$, 3rd, and 4 th blocks were paired with LeakyReLU activation. The patchGAN discriminator consisted of $44 \times 4$ convolutional kernels paired with instance normalization.

\section{Training, and Hypothesis Testing}

The model was trained using five different loss functions. The generator loss and L1 loss enforce spatial congruence between the input and output network signals. The Style loss uses loss from shallow and deep layers to help encourage stylistic consistency of the inpainted region. The VGG losses use deep networks to help enforce general realism of the predictions. The patchGAN discriminator loss helped preserve local texture features in the inpainted region. Figure 2 shows the generator, L1, Style, VGG, and discriminator loss functions.

The model was trained on 80,326 images and validated on 12,901 images. An additional 10,687 images corresponding to 40,077 ground truth CAL measurements were withheld from the data science team and used only once to avoid multiple hypothesis testing. Ground truth radiographic images were provided to blinded data scientists without clinical data for CAL predictions.

During inferencing, the discriminators were discarded and only the generators were used. The resulting inpainted image from the generator was fed into a CAL prediction. The two CAL prediction algorithms used in this study based on two open-source algorithms, Deep Lab (https://github.com/tensorflow/models/tree/master/research/deeplab) and DETR (https://github.com/facebookresearch/detr). We refer to Deep Lab as Method 1 and DETR as Method 2. The accuracy of the inpainted algorithm for method 1 (Inpaint 1) was compared to the non-inpainted algorithm for method 1 (Non-Inpaint 1) and the accuracy of the inpainted algorithm for method 2 (Inpaint 2) was compared to the non-inpainted algorithm for method 2 (Non-Inpaint 2).

The purpose of this study was to demonstrate the affect inpainting using various open-source CAL prediction algorithm. We could not replicate all foreseeable CAL prediction algorithms so only common open-source algorithms were used. 
To determine the affect inpainting had on the resulting CAL accuracy, MAE between the ground truth and predicted values was determined as well as comparator $p$-values from the Kruskal-Wallis test.

Additionally, pairwise differences in accuracy between each method was assessed using Dunn's pairwise comparison. The Dunn's test for pairwise comparisons was used as a post hoc test and adjusted for familywise error rate. Prediction accuracy among teeth (first molar, second molar, and premolar) was evaluated using the Kruskal-Wallis test. All hypothesis testing assumed a standard significance level of 0.05 .

\section{Results}

Figure 2 shows that the generator loss and discriminator loss converged at about 0.8 million iterations while the Style loss, VGG loss, and L1 loss converged at about 8.5 million iterations. The peak signal to noise ratio (PSNR) and structure similarity index measure (SSIM) continued to improve up to 8.5 million iterations. The model was stopped early to mitigate overfitting.

Figure 3. Representative images of method 1 without inpainting (left) and with inpainting (right). The left images show the original $x$-rays, and the right images show the inpainted $x$-rays with apical bone and teeth contours. The points represent the CEJ and apical bone positions representing CAL and the lines align the original and inpainted images.

Table 1 shows the mean absolute error (MAE), corresponding standard deviation (sdMAE), mean bias error (MBE), corresponding standard deviation (sdMBE), and sample size ( $n$ ) for all methods. The difference between Inpaint 1 and Non-Inpaint 1 was $0.46 \mathrm{~mm}$ MAE. Likewise, the difference between Inpaint 2 and Non-Inpaint 2 was $0.51 \mathrm{~mm}$ MAE.

Table 1

The mean absolute error (MAE), corresponding standard deviation (sdMAE), mean bias error (MBE), corresponding standard deviation (sdMBE), and sample size $(\mathrm{n})$ are shown for all methods.

\begin{tabular}{|llllll|}
\hline Measure & MAE & sdMAE & MBE & sdMBE & $\mathbf{n}$ \\
\hline Non-Inpaint 1 & 1.50 & 1.10 & -1.26 & 1.37 & 40,077 \\
\hline Non-Inpaint 2 & 1.66 & 1.18 & -1.43 & 1.45 & 40,077 \\
\hline Inpaint 1 & 1.04 & 0.86 & -0.43 & 1.28 & 40,077 \\
\hline Inpaint 2 & 1.15 & 0.92 & -0.58 & 1.36 & 40,077 \\
\hline Chi-squared (K-W) 3 df & 8955.74 & & & & \\
\hline P-value & $<0.0001$ & & & & \\
\hline
\end{tabular}

Table 2 shows the Dunn's pairwise comparison and corresponding p-values for all possible combinations of methods. A statistically significant Dunn's pairwise test value of -63.89 was observed between Inpaint 
1 and Non-Inpaint 1. Similarly, a statistically significant Dunn's pairwise test value of -65.20 was also observed between Inpaint 2 and Non-Inpaint 2.

Table 2

The Dunn's pairwise comparison and corresponding p-values are shown for all possible combinations of methods.

\begin{tabular}{|lll|}
\hline Comparison & Dunn's pairwise & p-value \\
\hline Inpaint 1 vs Inpaint 2 & -16.98 & $<0.0001$ \\
\hline Inpaint 1 vs Non-Inpaint 1 & -63.89 & $<0.0001$ \\
\hline Inpaint 1 vs Non-Inpaint 2 & -82.18 & $<0.0001$ \\
\hline Inpaint 2 vs NonInpaint 1 & -46.91 & $<0.0001$ \\
\hline Inpaint 2 vs NonInpaint 2 & -65.2 & $<0.0001$ \\
\hline NonInpaint 1 vs NonInpaint 2 & -18.29 & $<0.0001$ \\
\hline
\end{tabular}

Table 3 shows the sample size ( $n$ ), mean absolute error (MAE), corresponding standard deviation (sdMAE), mean bias error (MBE) and corresponding standard deviation (sdMBE) for the Premolar, First Molar and Second Molar teeth for all algorithms. 
Table 3

Sample size (n), mean absolute error (MAE), corresponding standard deviation (sdMAE), mean bias error (MBE), corresponding standard deviation (sdMBE), Chi-squared Kruskal-Wallis with 2 degrees of freedom and $p$ value, for tooth category, for all methods.

\begin{tabular}{|c|c|c|c|c|c|c|c|c|}
\hline \multirow[t]{2}{*}{ Method } & \multirow[t]{2}{*}{$\begin{array}{l}\text { Tooth } \\
\text { Category }\end{array}$} & \multirow[t]{2}{*}{$\mathrm{n}$} & \multirow[t]{2}{*}{ MAE } & \multirow[t]{2}{*}{ sdMAE } & \multirow[t]{2}{*}{ MBE } & \multirow[t]{2}{*}{ sdMBE } & $\begin{array}{l}\text { Chi-squared } \\
(\mathrm{K}-\mathrm{W})\end{array}$ & \multirow[t]{2}{*}{ p-value } \\
\hline & & & & & & & $2 \mathrm{df}$ & \\
\hline \multirow{3}{*}{$\begin{array}{l}\text { Non- } \\
\text { Inpaint } 1\end{array}$} & Premolar & 22246 & 1.40 & 1.01 & -1.15 & 1.28 & & \\
\hline & First Molar & 8899 & 1.61 & 1.09 & -1.40 & 1.34 & & \\
\hline & $\begin{array}{l}\text { Second } \\
\text { Molar }\end{array}$ & 8932 & 1.64 & 1.30 & -1.37 & 1.58 & 419.6 & $<0.0001$ \\
\hline \multirow{3}{*}{$\begin{array}{l}\text { Non- } \\
\text { Inpaint } 2\end{array}$} & Premolar & 22246 & 1.56 & 1.09 & -1.32 & 1.37 & & \\
\hline & First Molar & 8899 & 1.77 & 1.17 & -1.57 & 1.43 & & \\
\hline & $\begin{array}{l}\text { Second } \\
\text { Molar }\end{array}$ & 8932 & 1.80 & 1.36 & -1.54 & 1.65 & 1451.22 & $<0.0001$ \\
\hline \multirow[t]{3}{*}{ Inpaint 1} & Premolar & 22246 & 0.92 & 0.74 & -0.30 & 1.14 & & \\
\hline & First Molar & 8899 & 1.10 & 0.82 & -0.67 & 1.20 & & \\
\hline & $\begin{array}{l}\text { Second } \\
\text { Molar }\end{array}$ & 8932 & 1.30 & 1.08 & -0.51 & 1.61 & 1047.45 & $<0.0001$ \\
\hline \multirow[t]{3}{*}{ Inpaint 2} & Premolar & 22246 & 1.03 & 0.81 & -0.45 & 1.23 & & \\
\hline & First Molar & 8899 & 1.23 & 0.90 & -0.82 & 1.29 & & \\
\hline & $\begin{array}{l}\text { Second } \\
\text { Molar }\end{array}$ & 8932 & 1.41 & 1.13 & -0.66 & 1.68 & 889.89 & $<0.0001$ \\
\hline
\end{tabular}

\section{Discussion}

This study implemented a generative adversarial network inpainting method with partial convolutions to enhance the prediction of clinical attachment level compared to non-inpainted methods. Moreover, this is the first-time GAN-based inpainting was used to enhance the prediction of anatomical information in dentistry.

Table 1 demonstrated that inpainting methods consistently outperform non-inpainting methods for MAE and MBE. Correspondingly, Table 2 showed that all inpainting methods compared to non-inpainting methods were able to achieve statistically significant improvement in MAE using the Dunn's pairwise comparison. 
Figure 2 suggests that the model was stopped early and might have benefited from additional training. However, it took 135.2 hours to achieve approximately 8.5 million iterations using 8 V100 GPUs, so practical limitations and concerns about overfitting determined our iteration time. Also, the learning rate annealing was modulated to target a training duration of about 8.5 million iterations.

Overall, GAN inpainting was able to achieve more accurate CAL predictions compared to non-inpainting methods. The partial convolutions helped the model focus on relevant anatomy by limiting the influence of each layer to a sequestered anatomical region. The additional stylistic discriminator helped maintain the correct texture of boney mandibular and maxillary structures while preserving the subtle gingival soft tissue.

This study demonstrated a novel computer vision methodology applied to clinical attachment level prediction. In the interpretation of the statistical analysis, we found the mean absolute error to be informative. Clinician measured recession plus pocket depth determines the clinical attachment level. Clinician measurements are generally considered to be accurate plus or minus $1 \mathrm{~mm}$. We found the GAN inpaint method to be approximately within the clinical $1 \mathrm{~mm}$ accuracy standard, which gives us confidence that this method can be useful in predictions of CAL that are clinically meaningful and relevant.

This study employed commonly used bitewing and periapical radiographs. The methods used accounted for radiographic image distortion (foreshortening and elongation) which are common problems in measuring dental radiographic images. The techniques developed also accounted for angular bone differences making predications on the most apical bone levels. Clinician measured CAL is recorded in $1 \mathrm{~mm}$, whole number increments. The novel inpainting methods allowed for very accurate predictions of CAL that related within the plus or minus $1 \mathrm{~mm}$ clinical measurement standard. Cone beam CT (CBCT) and panoramic images were not evaluated in this study. Prior studies predicted periodontal bone loss from panoramic radiographs (13-15). However, panoramic and CBCT images tend to have a broader field of view and would not benefit from inpainting. Additionally, CBCT and panoramic images are acquired with routine resolutions far courser than bitewing or panoramic images and the larger source to detector distance of panoramic and CBCT images attribute to greater tissue scatter, more noise, more attenuation, higher $\mathrm{kVp}$, and poorer tissue contrast.

This study utilized purposive sampling of clinical data to provide validated findings, diagnosis with appropriate periodontal treatment, all in alignment. The patient clinical data used in this study was collected in a systematic manner within a dental institution. Educational and administrative systems are in place so that providers are calibrated, data are reviewed and approved by general dental and periodontal faculty where standards of care are adhered to and audited. These institutional quality control procedures assure high clinical data integrity. CAL measurements that were negative (gingival hypertrophy) and those of $6 \mathrm{~mm}$ and above (severe periodontitis) were not included in this study to help maintain the integrity of the ground truth measurements. We believe that these methods mitigate the "noise" that includes confirmation bias from data obtained from private dental practices. The purposive 
sampling used in this study followed methods utilized in the development, implementation and validation of clinical decision support, dental diagnostic terminology, quality measures, process of care, appropriateness of care and outcomes of care measurements(22-28).

\section{Conclusion}

We demonstrated the superiority of a novel generative adversarial network for CAL prediction on synthetic anatomy. GAN inpainting with partial convolutions and stylistic preservation presents a solution to overcome the challenges of CAL prediction in the presence of missing or diverse patient anatomy.

\section{Declarations}

\section{Author contributions}

V.K. and A.S. conceived the experiment. A.Y. conducted the statistical analysis and contributed to the study design. V.K., H.H., and W.D. coded the neural network. J.W., R.V., G.L. provided clinical ground truthing and contributed importance ideas to the study, R.B. prepared the data and contributed several key concepts to the study. N.J. and H.B. assisted in data collection. Everyone reviewed the manuscript.

\section{Competing Interests}

Neha Joshi, Harsh Bhandari and Joel White received support from an unrestricted gift of the Sue Bloch and Igor Khandros Family Foundation. Joel White received support from the Raymond L. and Mary V. Bertolotti Distinguished Professorship in Restorative Dentistry. Conflict of interest statement: Retrace investigators, Vasant Kearney, Hamid Hekmatian, Wenxiang Deng and Ali Sadat, are employees of Retrace. UCSF investigators, Alfa-Ibrahim Yansane, Ram Vaderhobli, Guo-Hao Lin, Neha Joshi, Harsh Bhandari, Joel White and Ryan Brandon, are employees or affiliates of UCSF and have no financial interests in Retrace and have no conflicts of interest. Vasant Kearney, Alfa Yansane, Ryan Brandon, Ram Vaderhobli, Guo-Hao Lin, Ali Sadat and Joel White contributed to conception and design and acquisition, analysis and interpretation. Hamid Hekmatian and Wenxiang Deng contributed to design and acquisition and analysis. Neha Joshi and Harsh Bhandari contributed to conception and design. All authors drafted the manuscript, critically revised the manuscript, gave final approval and agree to be accountable for all aspects of the work. This study was supported by a research grant to UCSF provided by Retrace Labs, Incorporated (San Francisco, CA. https://retrace.ai/).

\section{References}

1. Papapanou PN, Sanz M, Buduneli N, Dietrich T, Feres M, Fine DH, et al. Periodontitis: Consensus report of workgroup 2 of the 2017 World Workshop on the Classification of Periodontal and PeriImplant Diseases and Conditions. J Periodontol. 2018;89 Suppl 1:S173-s82. Epub 2018/06/22. doi: 10.1002/jper.17-0721. PubMed PMID: 29926951. 
2. Hirschfeld L, Wasserman B. A long-term survey of tooth loss in 600 treated periodontal patients. J Periodontol. 1978;49(5):225-37. Epub 1978/05/01. doi: 10.1902/jop.1978.49.5.225. PubMed PMID: 277674.

3. Needleman I, Garcia R, Gkranias N, Kirkwood KL, Kocher T, lorio AD, et al. Mean annual attachment, bone level, and tooth loss: A systematic review. J Periodontol. 2018;89 Suppl 1:S120-s39. Epub 2018/06/22. doi: 10.1002/jper.17-0062. PubMed PMID: 29926956.

4. Löe $H$, Anerud A, Boysen $H$, Morrison E. Natural history of periodontal disease in man. Rapid, moderate and no loss of attachment in Sri Lankan laborers 14 to 46 years of age. J Clin Periodontol. 1986;13(5):431-45. Epub 1986/05/01. doi: 10.1111/j.1600-051x.1986.tb01487.x. PubMed PMID: 3487557.

5. Arifin AZ, Indraswari R, Suciati N, Astuti ER, Navastara DA. Region merging strategy using statistical analysis for interactive image segmentation on dental anoramic readiographs. Int Rev Comput Softw. 2017;12(1):63.

6. Kearney V, Chen S, Gu X, Chiu T, Liu H, Jiang L, et al. Automated landmark-guided deformable image registration. Phys Med Biol. 2015;60(1):101-16. Epub 2014/12/06. doi: 10.1088/00319155/60/1/101. PubMed PMID: 25479095.

7. Barnes C, Shechtman E, Finkelstein A, Goldman DB. PatchMatch: A randomized correspondence algorithm for structural image editing. ACM Trans Graph. 2009;28(3):24.

8. Kearney V, Chan JW, Haaf S, Descovich M, Solberg TD. DoseNet: a volumetric dose prediction algorithm using 3D fully-convolutional neural networks. Phys Med Biol. 2018;63(23):235022. Epub 2018/12/05. doi: 10.1088/1361-6560/aaef74. PubMed PMID: 30511663.

9. Kearney V, Haaf S, Sudhyadhom A, Valdes G, Solberg TD. An unsupervised convolutional neural network-based algorithm for deformable image registration. Phys Med Biol. 2018;63(18):185017. Epub 2018/08/16. doi: 10.1088/1361-6560/aada66. PubMed PMID: 30109996.

10. Ronneberger O, Fischer P, Brox T. U-net: Convolutional networks for biomedical image segmentation. International Conference on Medical iage computing and computer-assisted intervention: Pringer, Cham; 2015.

11. Kearney V, Chan JW, Descovich M, Yom SS, Solberg TD. A multi-task CNN model for autosegmentation of prostate patients. International Journal of Radiation Oncology, Biology and Physics. 2018;102(3):S214.

12. Kearney V. A Novel Semantic CT Segmentation Algorithm Using Boosted Attention-Aware Convolutional Neural Networks. 111 River Street, Hoboken, NJ: Wiley; 2019.

13. Krois J, Ekert T, Meinhold L, Golla T, Kharbot B, Wittemeier A, et al. Deep Learning for the Radiographic Detection of Periodontal Bone Loss. Sci Rep. 2019;9(1):8495. Epub 2019/06/13. doi: 10.1038/s41598-019-44839-3. PubMed PMID: 31186466; PubMed Central PMCID: PMCPMC6560098.

14. Kim J, Lee HS, Song IS, Jung KH. DeNTNet: Deep Neural Transfer Network for the detection of periodontal bone loss using panoramic dental radiographs. Sci Rep. 2019;9(1):17615. Epub 
2019/11/28. doi: 10.1038/s41598-019-53758-2. PubMed PMID: 31772195; PubMed Central PMCID: PMCPMC6879527 no potential conflict of interest.

15. Chang HJ, Lee SJ, Yong TH, Shin NY, Jang BG, Kim JE, et al. Deep Learning Hybrid Method to Automatically Diagnose Periodontal Bone Loss and Stage Periodontitis. Sci Rep. 2020;10(1):7531. Epub 2020/05/07. doi: 10.1038/s41598-020-64509-z. PubMed PMID: 32372049; PubMed Central PMCID: PMCPMC7200807.

16. Goodfellow I, Pouget-Abadie J, Mirza M, Xu B, Warde-Farley D, Ozair S, et al. Generative adversarial nets. Advances in neural information processing systems. 2014: 2672-80.

17. Kearney V, Ziemer BP, Perry A, Wang T, Chan JW, Ma L, et al. Attention-Aware Discrimination for MRto-CT Image Translation Using Cycle-Consistent Generative Adversarial Networks. Radiology: Artificial Intelligence. 2020;2(2):e190027.

18. Kearney V, Chan JW, Wang T, Perry A, Descovich M, Morin O, et al. DoseGAN: a generative adversarial network for synthetic dose prediction using attention-gated discrimination and generation. Sci Rep. 2020;10(1):11073. Epub 2020/07/08. doi: 10.1038/s41598-020-68062-7. PubMed PMID: 32632116; PubMed Central PMCID: PMCPMC7338467.

19. Kearney V, Zeimer BP, Perry A, Wang T, Chan JW, Yom SS, et al. Spatial Attention Gated Variational Autoencoder Enhanced Cycle-Consistent Generative Adversarial Networks for MRI to CT Translation. International Journal of Radiation Oncology, Biology, Physics. 2019;1(1051):E720-E1.

20. Liu G, Reda FA, Shih KJ, Wang TC, Tao A, Catanzaro B. Image inpainting for irregular holes using partial convolutions. Proceedings of the European Conference on Computer Vision (ECCV), pp 851002018. p. pp. 85-100.

21. Kearney V, Sadat A, Chan S, Hakmatian H, Patel Y, inventors; Retrace Labs Inc, assignee. Artificial Intelligence Architecture for Identification of Periodontal Features. patent US20200364860A1. November 19, 2020.

22. Kalenderian E, Ramoni RL, White JM, Schoonheim-Klein ME, Stark PC, Kimmes NS, et al. The development of a dental diagnostic terminology. J Dent Educ. 2011;75(1):68-76. Epub 2011/01/06. PubMed PMID: 21205730; PubMed Central PMCID: PMCPMC3107733.

23. White JM, Kalenderian E, Stark PC, Ramoni RL, Vaderhobli R, Walji MF. Evaluating a dental diagnostic terminology in an electronic health record. J Dent Educ. 2011;75(5):605-15. Epub 2011/05/07. PubMed PMID: 21546594; PubMed Central PMCID: PMCPMC3119719.

24. Tokede $\mathrm{O}$, White J, Stark PC, Vaderhobli R, Walji MF, Ramoni R, et al. Assessing use of a standardized dental diagnostic terminology in an electronic health record. J Dent Educ. 2013;77(1):24-36. Epub 2013/01/15. PubMed PMID: 23314462; PubMed Central PMCID: PMCPMC3545411.

25. Yansane A, Tokede O, White J, Etolue J, McClellan L, Walji M, et al. Utilization and Validity of the Dental Diagnostic System over Time in Academic and Private Practice. JDR Clin Trans Res. 2019;4(2):143-50. Epub 2019/04/02. doi: 10.1177/2380084418815150. PubMed PMID: 30931711.

26. Kumar SV, Bangar S, Neumann A, Kookal KK, Yansane A, Tokede O, et al. Assessing the validity of existing dental sealant quality measures. J Am Dent Assoc. 2018;149(9):756-64.e1. Epub 
2018/07/08. doi: 10.1016/j.adaj.2018.05.001. PubMed PMID: 29980245.

27. White JM, Mertz EA, Mullins JM, Even JB, Guy T, Blaga E, et al. Developing and Testing Electronic Health Record-Derived Caries Indices. Caries Res. 2019(53):650-8. Epub 2019/06/06. doi:

10.1159/000499700. PubMed PMID: 31167186.

28. White JM, Brandon RG, Mullins JM, Simmons KL, Kottek AM, Mertz EA. Tracking oral health in a standardized, evidence-based, prevention-focused dental care system. J Public Health Dent. 2020;80(S2):S35-S43. Epub 2020/10/27. doi: 10.1111/jphd.12413. PubMed PMID: 33104245.

\section{Figures}

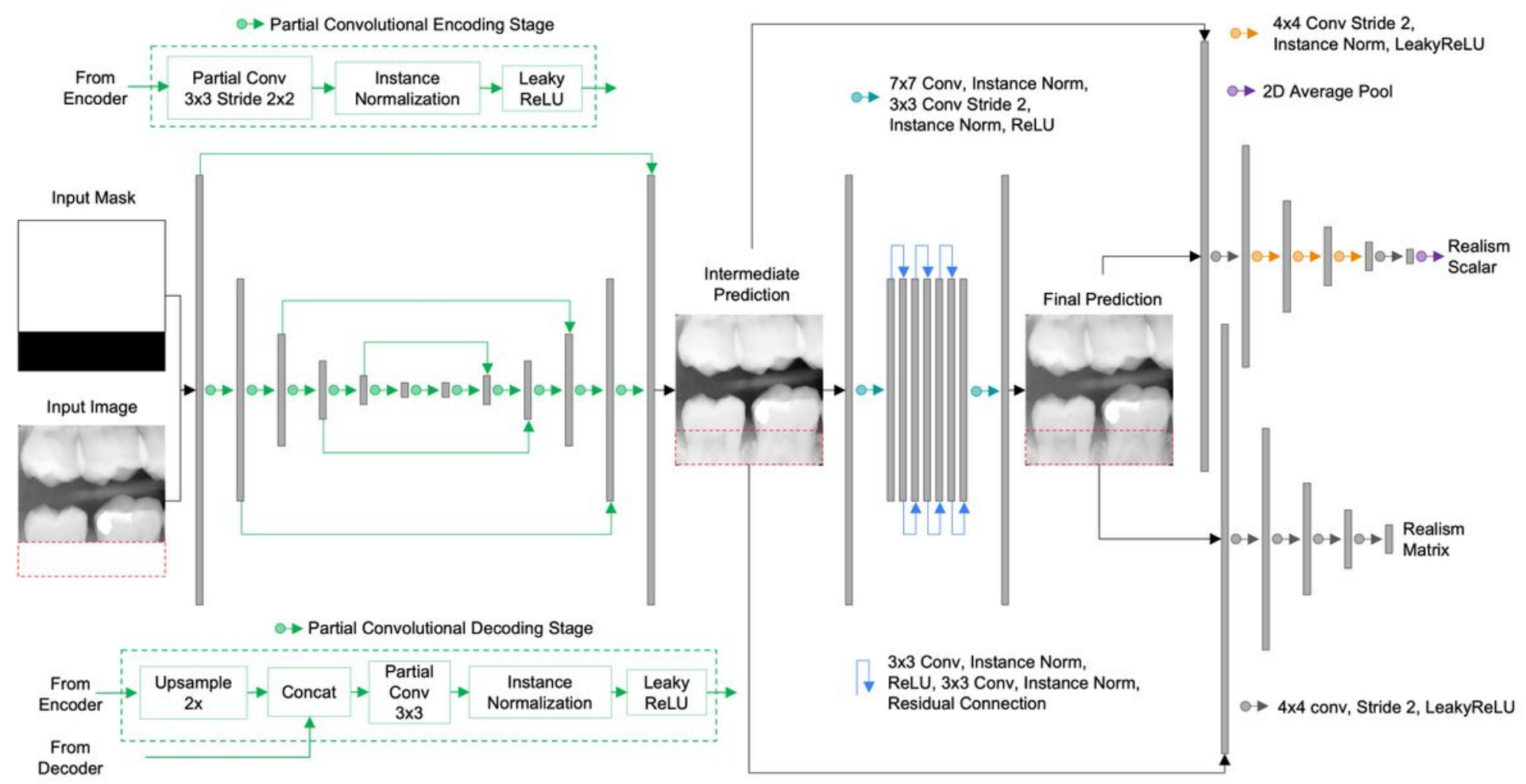

Figure 1

The Partial Convolutional Encoder-Decoder generator, Refine Encoder-Decoder generator, VGG discriminator, and patchGAN discriminator networks are shown as well as the network subcomponents. 
Generator

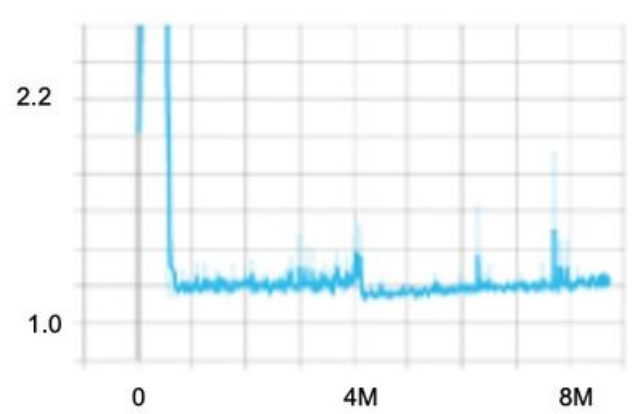

L1 Loss

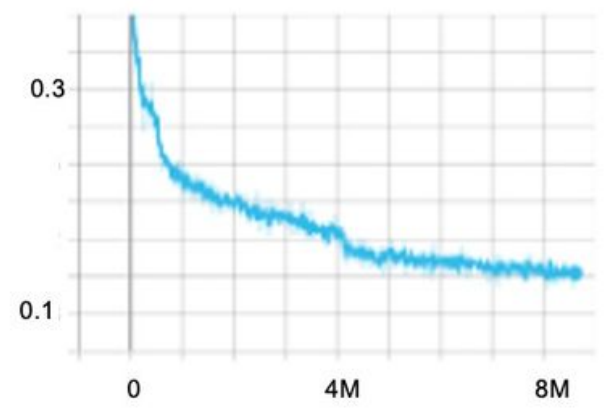

Style Loss

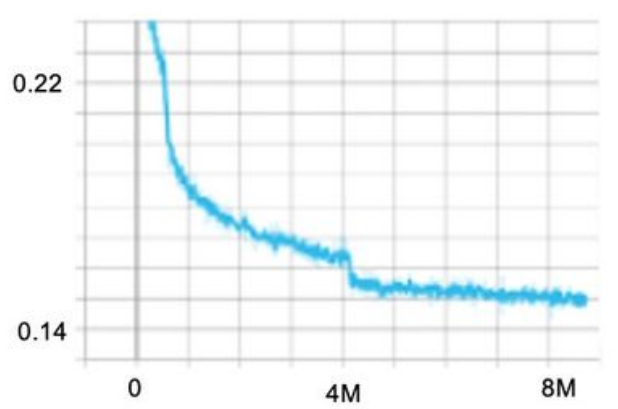

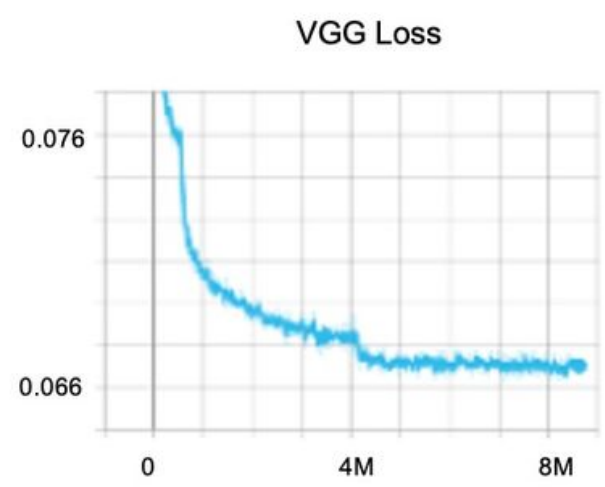

VGG Loss

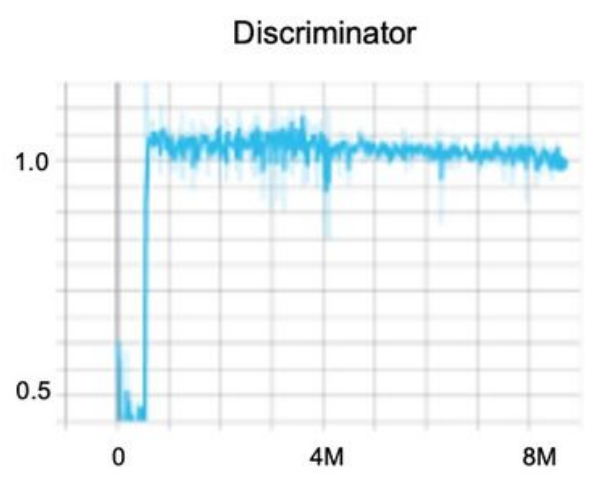

\section{Figure 2}

The generator, L1, Style, VGG, and discriminator loss functions are shown for each iteration throughout the training process. 


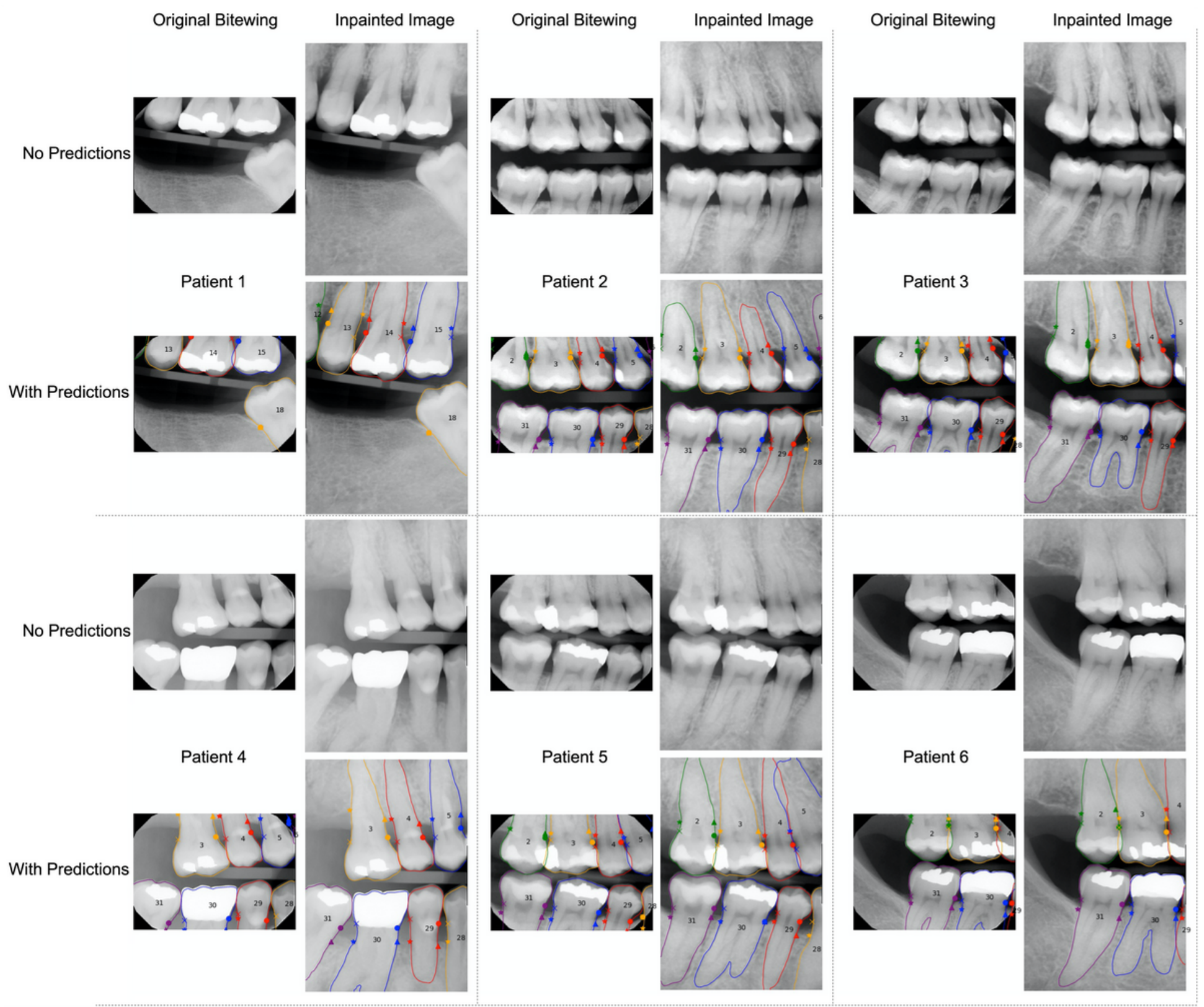

Figure 3

Representative images of method 1 without inpainting (left) and with inpainting (right). The left images show the original $x$-rays, and the right images show the inpainted $x$-rays with apical bone and teeth contours. The points represent the CEJ and apical bone positions representing CAL and the lines align the original and inpainted images. 